\title{
New Revolutionary Hybrid SEM by Carl Zeiss
}

\author{
Mehdi Bolorizadeh, Friedhelm Panteleit ${ }^{*}$, Frank F. Hitzel ${ }^{\dagger}$ \\ Carl Zeiss Microscopy, LLC; 1Zeiss Dr., Thornwood, NY 10594, USA \\ * Carl Zeiss Microscopy, GmbH; Carl-Zeiss-Straße 22, 73447 Oberkochen, Germany \\ ${ }^{\dagger}$ DME Nanotechnologie GmbH; Geysostraße 13, D-38106 Braunschweig, Germany
}

At Microscopy \& Microanalysis conference 2012 in Phoenix, USA, Carl Zeiss Microscopy introduced MERLIN Compact and MERLIN Variable Pressure (VP) Compact, which will complement the existing MERLIN platform. The modular design of the Merlin platform enables the customer to exactly tailor the two new products', MERLIN (VP) Compact, configuration to each customer's application. Even complex application solutions (e.g. SEM-3View block sectioning by Gatan [1], SEM-AFM by DME [2]) can be realized by this flexibility. This abstract will mainly emphasize on the hybrid MERLIN (VP) Compact-AFM.

The company DME produces the AFM option exclusively for Carl Zeiss (CZ) and provides full application support to the $\mathrm{CZ}$ team and to potential customers. DME is one of the first and thereby most experienced Scanning Probe Microscopy manufacturers in the world. The CZ SEM-AFM hybrid technology enables the customer access to new application areas and exceptional research possibilities. Combined measurements, opens access to information which cannot be obtained by one of the methods alone (e.g. tip-induced voltage contrast on nanometer scale). With atomic resolution in all 3 dimensions, the AFM delivers information such as topography, magnetic, mechanical and surface potential, element distribution and analysis, and material structure (FIG 1). SEM users benefit from calibrated 3D topography information (FIG 2), AFM based nano-manipulation and modification, and Access to mechanical and electrical sample information. AFM users benefit from the large magnification range of the SEM and its faster scanning speed (25ns/pixel) to select an ROI for AFM investigation.

The CZ SEM-AFM hybrid system makes surface manipulation live on screen possible with all available AFM cantilever types [3]. The MERLIN SEM-AFM hybrid is the only hardware- and software integrated system on the market of its kind. With simple tip and sample exchange through airlock and an integrated user interface the SEM-AFM hybrid follows the ease of use principle of the new MERLIN series. The Zeiss SEM/AFM hybrid gives access to new customer target groups. With the hybrid system it is possible to reach customers which are mainly interested in AFM related equipment and which require straight forward obtainable atomic height information combined with real-time AFM tip observation and positioning. This hybrid system can be operated as a Magnetic Force Microscope (MFM) to study the magnetic properties of a material. This mode can be used in multiphase magnetic materials such as the powder steel sample in FIG 3 in which the magnetic image clearly shows the boundaries of austenite (non-magnetic) and ferrite (magnetic) phases in the material.

The MERLIN series is optimized to integrate such advanced application solutions. The 2012 launched MERLIN (VP) Compact products complete the MERLIN product family and take over many advanced features from MERLIN, but stay at the same price level as predecessor products. For the VP version two different charge compensation (CC) methods are available in one tool for the whole beam energy range (CC and VP) leading to improved nano-analytics of non-conductive samples (and no time consuming sample preparation of non-conductive samples). The new InlensDuo detector gives 
customers with lower budget the possibility to benefit from the unique low voltage Energy Selective Backscatter (EsB) detector contrasts, which is a powerful tool for material with poor low voltage contrasts and was well received by the EM community. At least but not at last the fast, low noise, forward-design SEM electronics is ready for future technology integration, for example for easily upgradable plug \& play detector solutions.

\section{References:}

[1] http://www.gatan.com

[2] http://www.dme-spm.com

[3] http://microscopy.zeiss.com/microscopy/en_de/products/electron-microscopy/merlin-materials.html

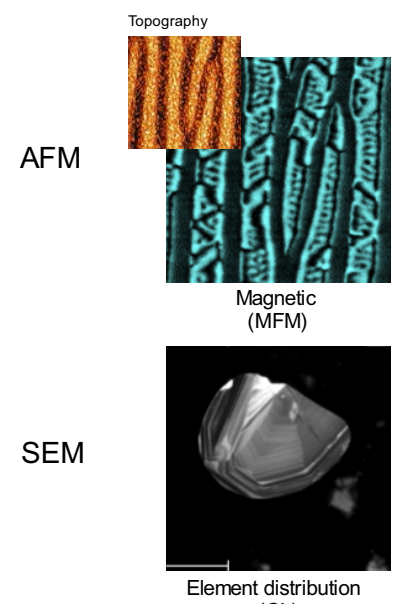

$(\mathrm{CL})$

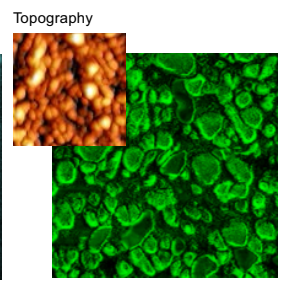

Surface potential (KPFM)

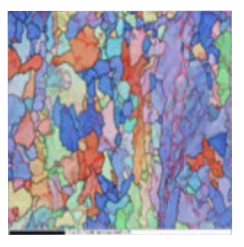

Material structure (EBSD)

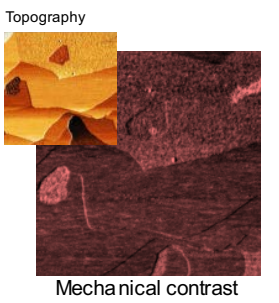

(Phase)

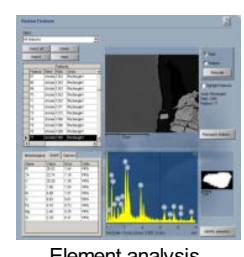

Element analysis

FIG. 1. A selection of accessible sample information from a hybrid SEM-AFM system.

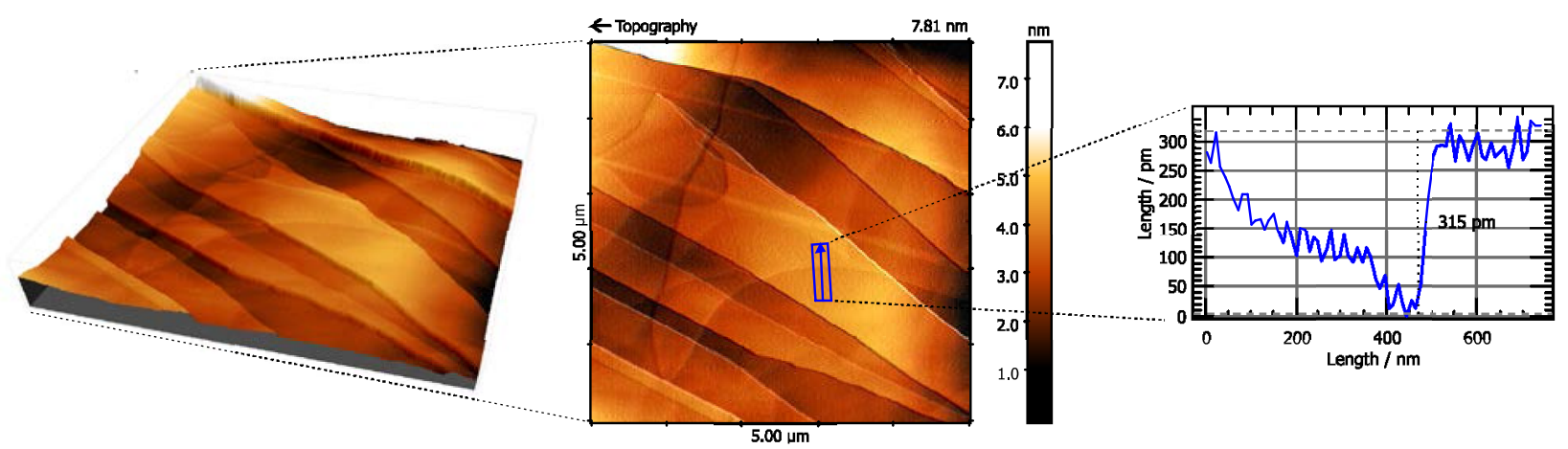

FIG. 2. 3D and 2D plots of a Highly Ordered Pyrolytic Graphite (HOPG) and a 2D graph showing a single atomic step of a graphene layer in the HOPG.
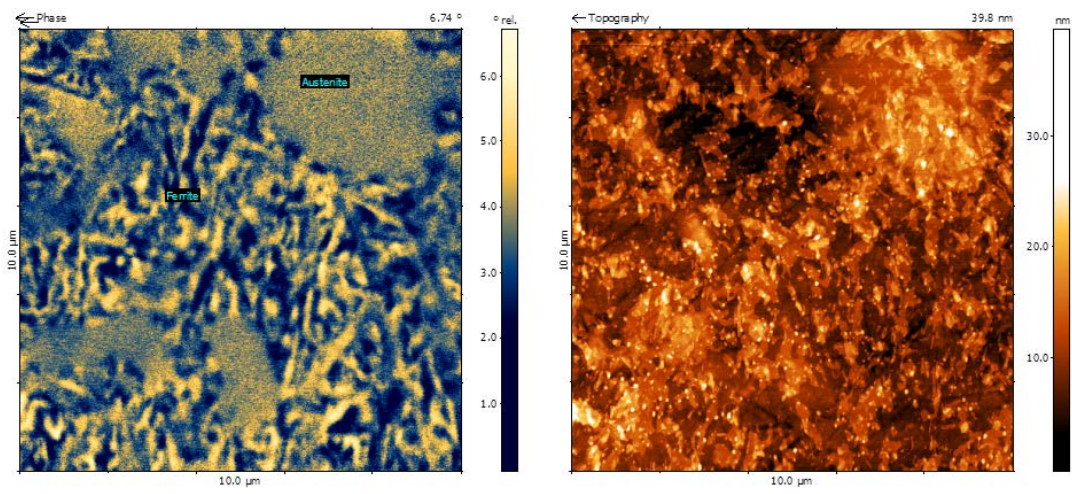

FIG. 3. Magnetic and topographic images of a powder steel sample. In the magnetic image, the austenite and ferrite regions are clearly distinguishable. 